\title{
From Leather Bags to Webcams, the Emerging Tools of Tele-primary Care
}

\author{
William B. Smith II, MD, MPH, MBA ${ }^{1,2}$ and R. Jeffrey Koh/wes, MD, MPH ${ }^{1,2}$ \\ 'Veterans Affairs Medical Center, San Francisco, CA, USA; ${ }^{2}$ Department of Medicine, University of California, San Francisco, San Francisco, CA, \\ USA.
}

J Gen Intern Med 35(3):628-9

DOI: $10.1007 / \mathrm{s} 11606-019-05603-5$

(C) Society of General Internal Medicine (This is a U.S. government work and not under copyright protection in the U.S.; foreign copyright protection may apply) 2019

F or many years, the physician's leather bag has been an essential component of the primary care physician's armamentarium - used for holding everything needed for house calls and symbolizing the physician's craft. As the availability and technology of telemedicine increases, the leather bag is expanding to include a laptop and webcam. Currently, 79 million Americans live in Health Professional Shortage Areas (HPSAs), ${ }^{1}$ and healthcare costs the USA \$3.5 trillion annually. Therefore, innovations, such as telehealth, with the potential to deliver high-quality care to greater numbers at lower cost must be further explored. While optimal scope and structure are yet to be determined, high-quality telemedicine care is an opportunity to reach more patients and should stretch beyond urgent care to become an important tool for primary care providers.

The average patient spends more than 2 hours for every medical appointment between time spent traveling and waiting in the office. ${ }^{2-4}$ Virtual visits eliminate travel, minimize time away from work, and may improve patient experience with immediate care in the privacy of their own homes. Private telemedicine is available to anyone with internet and a smart phone. From the perspective of an individual patient, per-visit co-pays are substantially lower than urgent care alternatives, and visits for the uninsured can frequently be found for less than $\$ 50$ per consultation. ${ }^{5}$ Telemedicine companies often have little overhead, cover massive catchment areas, and employ staff for $24 / 7$ access.

Despite the promise of telemedicine, there is a paucity of data demonstrating consistently high-quality care. Rastogi and colleagues make an important contribution to the literature by characterizing a large direct-to-consumer (DTC) telemedicine system's management of a common condition: urinary tract infection. ${ }^{6}$ Their analysis showed high-quality care for average risk women, demonstrated by largely appropriate antibiotic prescribing, short visits, and high patient satisfaction. The work is also notable because of the questions it

Published online January 2, 2020 raises for future investigation including management of high-risk patients, linkage of patient satisfaction to prescribing practices, record sharing across systems/regions, and the impact of care fragmentation.

There is little debate over the safety of remote assessment, by video or phone, of low-risk women complaining of UTI symptoms, particularly for those with a history of prior UTI. ${ }^{7}$ After all, 92\% of women in this sample whose chief complaint was "UTI" were diagnosed as having a UTI, consistent with results of previous self-diagnosis studies. Also, similar to what is observed across practice settings, a small cohort of providers represented the majority of guideline discordant antibiotic prescribing. As the authors note, this underscores the importance of integrated decision support systems to address variability in decision making for often algorithmic treatment.

Another issue raised by the authors was that management of high-risk patients was less consistent and their follow-up plans were unknown to primary care providers. High-risk patients represented $6 \%$ (1171) of the sample and were defined as men, women over 65 , and those diagnosed with pyelonephritis. These patients, mostly men $(63 \%)$, had no urine cultures and yet, received antibiotics $69 \%$ of the time. This data point is striking because in similar studies treating UTI diagnosis by telephone, high-risk patients have always been excluded. ${ }^{7}$ How is telephone different than telemedicine in these cases? Perhaps providers are emboldened by visualizing patients on screen, their image providing reassurance to prescribe for patients they otherwise would frame as high risk.

This is an example of where primary care providers with longitudinal patient relationships could facilitate appropriate triage. Patients with previously investigated diagnoses precipitating UTI, or recurrent UTI with clear prior culture results, might prompt a direct prescription without a visit to the clinic. Knowledge of medical history and ability to secure follow-up are requisite for safe remote management of high-risk patients. To treat these high-risk patients safely, prescribers should have knowledge of their medical history and clear coordination for follow-up care. These are attributes of primary care involvement in tele-healthcare.

Another argument for primary care entering the telehealth space is the lack of health record sharing and poor follow-up care coordination seen thus far in direct-to-consumer healthcare. This may increase the risk of medical errors, unnecessary testing, and costly inefficiencies. The average Medicare beneficiary 
utilizes four different practices and seven separate physicians. ${ }^{2}$ We cannot afford for this statistic to worsen. Literature on use of telemedicine for discrete conditions shows a trend toward overprescribing, generally less consistent guideline-concordant therapy, ${ }^{8-11}$ varying appropriateness of diagnostic testing, ${ }^{8,} 10-12$ and shorter follow-up intervals. ${ }^{10,12}$

This study and others suggest a relationship between receipt of a prescription and patient satisfaction. ${ }^{12}$ In crowded markets with consumers sensitive to price and proxy indicators of quality such as 5-star reviews, companies are incentivized to optimize these levers to increase demand. In this study, Rastogi et al. segmented review scores to 5-stars and less than 5 stars; $96 \%$ of respondents who received antibiotics gave a 5star review versus $85 \%$ of those who did not. We do not know what average review would prompt consumers to consider alternative service providers for a given telemedicine service. If potential patients can easily choose among a variety of competing DTC telemedicine companies, maximizing positive reviews becomes a powerful incentive that may at times conflict with guideline-concordant therapy. Subsequent analyses should further characterize this relationship.

Many of the access and continuity concerns are familiar as they were raised as retail clinics emerged in the early 2000 s. These included scope of practice, health record interoperability, redundant testing, and impact on overall societal cost. Both retail clinics and DTC telemedicine enterprises focused on acute care may contribute to an increase in per capita spending ${ }^{13}$ but their integration with large healthcare organizations and a shifting focus toward chronic disease management could change the equation.

Many retail clinics have expanded their scope of practice and partnered with large payers and health systems. CVS MinuteClinics and Walgreens "Take Care" clinics have individually partnered with large health systems (e.g., Cleveland Clinic, Ochsner Health in New Orleans, Henry Ford Health System). ${ }^{14}$ Through these partnerships, retail clinics are now managing costly chronic conditions including hypertension, diabetes, and asthma. Records are shared electronically, and retail clinics are empowered to secure follow-up appointments for patients at their usual primary care clinics. Such partnerships have similar potential to integrate DTC telemedicine into the medical home.

Telemedicine is well suited for more convenient and consistent management of chronic illnesses. The meteoric rise of DTC telemedicine and other convenience-focused services is a call to action for primary care. If shorter wait times, low outof-pocket costs, and reduced travel time are driving demand for these services, primary care practices should expand convenience-based offerings through partnership or by developing new capacity. Expanded services should be tailored both to at-risk HPSA populations as well as to those most likely to pursue care based on convenience, like the young-healthy urban cohort. ${ }^{9}$ DTC telemedicine should complement the medical home, allowing more frequent contact with patients and easier access. Embracing telemedicine offerings in primary care will mitigate many of the risks around record sharing, redundant testing, and quality control. When integrated with longitudinal primary care, telemedicine may facilitate better care team communication, better outcomes, and lower cost to the health system.

Corresponding Author: R. Jeffrey Kohlwes, MD, MPH; Department of Medicine University of California, San Francisco, San Francisco, CA, USA (e-mail: Jeff.Kohlwes@ucsf.edu).

\section{REFERENCES}

1. U.S. Department of Health \& Human Services, Health Resources and Services Administration (HRSA). Designated Health Professional Shortage Areas Statistics: Designated HPSA Quarterly Summary, as of December 31, 2018. 2018.

2. Liaw WR, Jetty A, Coffman M, et al. Disconnected: a survey of users and nonusers of telehealth and their use of primary care. J Am Med Inform Assn. 2019;26(5):420-428. doi:https://doi.org/10.1093/jamia/ocy182

3. Ray KN, Chari AV, Engberg J, Bertolet M, Mehrotra A. Disparities in Time Spent Seeking Medical Care in the United States. Jama Intern Med. 2015;175(12):1-3. doi:https://doi.org/10.1001/jamainternmed.2015.4468

4. Dorsey RE, Dorsey E, Topol EJ, Topol E. State of telehealth. New Engl J Medicine. 2016;375(2):154-161. doi:https://doi.org/10.1056/ nejmra1601705

5. Bell T. Can Telemedicine Be Both Cost Efficient and High Quality? US News \& World Report. 2018. Accessed 10/28/19 at https://www. usnews.com/news/healthcare-of-tomorrow/articles/2018-02-27/cantelemedicine-be-both-cost-efficient-and-high-quality

6. Rastogi R, Martinez KA, Gupta N, Rood M, Rothberg MB. Management of Urinary Tract Infections in Direct to Consumer Telemedicine. J Gen Intern Med. 2019. DOI: https://doi.org/10.1007/s11606-019-05415-7

7. Colgan R, Williams M. Diagnosis and treatment of acute uncomplicated cystitis. Am Fam Physician. 2011;84(7):771-776.

8. Ray KN, Shi Z, Gidengil CA, Poon SJ, Uscher-Pines L, Mehrotra A. Antibiotic Prescribing During Pediatric Direct-to-Consumer Telemedicine Visits. Pediatrics. 2019;143(5):e20182491 14. doi:https://doi.org/10. 1542/peds.2018-2491

9. Uscher-Pines L, Mulcahy A, Cowling D, Hunter G, Burns R, Mehrotra A. Access and Quality of Care in Direct-to-Consumer Telemedicine. Telemed E-health. 2016;22(4):282-287. doi:https://doi.org/10.1089/ tmj.2015.0079

10. Jain T, Lu RJ, Mehrotra A. Prescriptions on Demand. Jama. 2019;322(10):1-2. doi:https://doi.org/10.1001/jama.2019.9889

11. Shi Z, Mehrotra A, Gidengil CA, Poon SJ, Uscher-Pines L, Ray KN. Quality Of Care For Acute Respiratory Infections During Direct-ToConsumer Telemedicine Visits For Adults. Health Affair. 2018;37(12):20142023. doi:https://doi.org/10.1377/hlthaff.2018.05091

12. Foster CB, Martinez KA, Sabella C, Weaver GP, Rothberg MB. Patient Satisfaction and Antibiotic Prescribing for Respiratory Infections by Telemedicine. Pediatrics. 2019;144(3):e20190844. doi:https://doi.org/ 10.1542/peds.2019-0844

13. Ashwood SJ, Gaynor M, Setodji CM, Reid RO, Weber E, Mehrotra A. Retail Clinic Visits For Low-Acuity Conditions Increase Utilization And Spending. Health Affair. 2017;35(3):449-455. doi:https://doi.org/10. 1377/hlthaff.2015.0995

14. Dalen JE. Retail Clinics: A Shift From Episodic Acute Care to Partners in Coordinated Care. Am J Medicine. 2016;129(2):134-136. doi:https://doi. org/10.1016/j.amjmed.2015.08.019

Publisher's Note Springer Nature remains neutral with regard to jurisdictional claims in published maps and institutional affiliations. 